\title{
Acute renal damage in Acetaminophen poisoning
}

\author{
Faizan Mazhar ${ }^{1,2^{*}}$ and Shahzad Akram ${ }^{2}$ \\ 'Department of Basic Medical Sciences, Prince Sultan Military College of Health Sciences, King Fahad Military Medical Complex, Dhahran, SAUDI ARABIA. \\ 2Department of Pharmaceutical care, King Abdulaziz Medical City, National Guard Health Affairs, Riyadh, SAUDI ARABIA.
}

Correspondence : Faizan Mazhar, Department of basic medical sciences, Prince Sultan Military College of Health Sciences, King Fahad Military Medical Complex, Dhahran, SAUDI ARABIA. Phone no: 00966548235061 E-mail: mfznbaig@gmail.com DOI: 10.5530/jyp.2016.4.32

\section{Dear Sir,}

Acetaminophen is one of the most commonly used analgesic globally. It is first-line pharmacologic agent for the symptomatic treatment of mild to moderate pain recommended by WHO. Acetaminophen may act as a potent hepatotoxic and it is account for $5 \%$ of drug poisonings cases, being a frequent cause of acute liver failure. ${ }^{1}$ It is still little-known fact that acetaminophen poisoning can cause kidney injury, especially for those pharmacist who are at an early stage of clinical pharmacy career. Therefore this prompt us to provide two interesting cases of acetaminophen poisining with renal manifestions in which clinical pharmacists were active members of the care team.

\section{Case 1}

A 46-year-old woman made a suicide attempt by the ingestion $40 \mathrm{~g}$ of acetaminophen in a single dose. She was consulted to an emergency department after 15 hours of repeated vomiting. Plasma concentration of paracetamol was found to be $249 \mu \mathrm{g} / \mathrm{ml}$, aspartate transaminase (AST)/ alanine transaminase (ALT) levels were 431/432 IU, bilirubin $1.2 \mathrm{mg} / \mathrm{dl}$, prothrombin time $40 \%$. Renal function was preserved without metabolic acidosis. Treatment was started with $\mathrm{N}$-acetylcysteine, and the patient was transferred to the intensive care unit. Lab analysis is shown in Table 1. She remained clinically stable and showed no signs of hepatic encephalopathy. On hospital day fourth, there was a progressive rise in serum creatinine levels with positive markers of tubular necrosis, she developed oliguric renal failure for which she undergoes three sessions of haemodialysis up to the tenth day of hospitalization. The subsequent clinical course was uneventful, with complete restoration of hepatic and renal function.

\section{Case 2}

A 42-year-old man former heroin abuser, presents to the emergency room with a chief complaint of abdominal pain, nausea and vomiting for the past four days. He denies hematemesis, fever and continuing alcohol intake. His current medications includes paracetamol and lormetazepam as per needed for chronic lower back pain. The lab analysis on admission is shown in Table 1. Initially, he was diagnosed with food poisoning and admited to medicine department for fluid and antibiotic therapy. On following days he was markedly dehydrated with abnormal liver function test (LFT). On physical examination, he was a little tachycardic, tachypneic, and afebrile. Despite scheduled antiemetic treatment he vomited 3-4 times and developed left flank pain. On his laboratory examination, the most significant findings were a serum creatinine level of $10.29 \mathrm{mg} /$ $\mathrm{dl}$ and AST $739 \mathrm{IU} / \mathrm{l}$. His neurological examination was consistent with grade-ii hepatic encephalopathy. At this point his diagnosis was re-evaluated. On requisitioning the patient reported a consumption of $4 \mathrm{~g} /$ day of paracetamol in last few days for the exacerbation of back pain. Plasma paracetamol level was ordered, it was $22 \mu \mathrm{g} / \mathrm{ml}$. Treatment was initiated with $\mathrm{N}$-acetylcysteine, antibiotic prophylaxis, and primary prophylaxis for stress ulcers. Three haemodialysis sessions were performed for acute kidney dysfunction. There was a progressive clinical recovery of the patient without sequelae.

Although the main site of acetaminophen metabolism is liver, it is known that this drug can also cause direct renal damage from the studies carried out in hepatectomized mice and in-vitro kidney model, this may occur early in the course of the illness. In addition, paracetamol poisoning has been reported with renal injury without liver failure. and renal failure. The prognosis worsens when acute kidney injury concomitantly present

Table 1: Lab analysis

\begin{tabular}{|c|c|c|c|c|c|c|c|c|c|c|}
\hline \multirow{8}{*}{$\overline{\tilde{U}}$} & Days & Admission & 1 & 2 & 4 & 6 & 8 & 10 & 15 & 30 \\
\hline & AST (IU/1) & 1.033 & 1.754 & 15.75 & 522 & 79 & 57 & & 82 & 38 \\
\hline & $\operatorname{ALT}(\mathrm{IU} / \mathrm{l})$ & 1.123 & 2.714 & 14.854 & 3.675 & 1.954 & 743 & & 71 & 53 \\
\hline & Total Bilirubin (mg/dl) & 2.7 & 4.3 & 3.7 & 3.8 & 1.904 & 753 & & 72 & 59 \\
\hline & Prothrombin time (\%) & 22.3 & 33 & 16.9 & 43 & 61.4 & 58 & 90 & 94 & \\
\hline & Serum Creatinine (mg/ dl) & 0.4 & 0.57 & 2.06 & 6.42 & 9.34 & 6.34 & 5.45 & 2.86 & 0.74 \\
\hline & Glomerular filtration rate $(\mathrm{ml} / \mathrm{min})$ & $>85$ & $>85$ & 18 & 13 & 8 & 14 & 9.3 & 22.5 & 83 \\
\hline & Phosphorus (mg / dl) & 1.45 & & & & 9.33 & & & & 3.23 \\
\hline \multirow{8}{*}{ 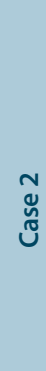 } & $\beta$-2-microalbumin in urine $(\mu \mathrm{g} / \mathrm{g} \mathrm{cr})$ & & & & 8.9743 & & & & & \\
\hline & AST (IU/1) & 2.349 & 739 & 318 & 134 & 96 & & 51 & 38 & 33 \\
\hline & ALT (IU/1) & 6.936 & 4.339 & 2.753 & 1.369 & 830 & 778 & 380 & 142 & 47 \\
\hline & Total Bilirubin (mg/dl) & 7.8 & 6.6 & 7.4 & 6.2 & 4.5 & 3.39 & 2.88 & 1.3 & 1.2 \\
\hline & Prothrombin time (\%) & 28.9 & 37.5 & 56.4 & 67.1 & 72.9 & 68 & 81 & 74 & 98 \\
\hline & Serum Creatinine (mg/ dl) & 7.43 & 10.29 & 6.8 & 5.4 & 5.19 & 4.78 & 4.34 & 1.34 & 1.22 \\
\hline & Glomerular filtration rate $(\mathrm{ml} / \mathrm{min})$ & 9.44 & 11.32 & 7.16 & 11.78 & 26.75 & 32.54 & 37.23 & 54.54 & $>90$ \\
\hline & Phosphorus (mg / dl) & & & 2.08 & & & & 5.78 & & 4.67 \\
\hline
\end{tabular}


with acute liver failure. ${ }^{2}$ The mechanism of acetaminophen-induced nephrotoxicity is still not clear. However, some authors relate this with the renal metabolism of acetaminophen, similar to the liver, with the formation of a reactive metabolite ( $\mathrm{N}$-acetyl-p-benzoquinoneimida [NAPQI]) which cause cell destruction mainly in tubules of the renal cortex when glutathione reserves depleted. ${ }^{3,4}$ Another hypothesis is the involvement of the prostaglandin endoperoxide synthetase enzyme in paracetamol metabolism which leads to the formation of NAPQI. Toxicity of acetaminophen at therapeutic doses could explain by the activation of NAPQI metabolic route via prostaglandin endoperoxide synthetase enzyme. A third hypothesis is related to $\mathrm{N}$-deacetylase enzyme, which generates p-aminophenol and this, in turn, generates free radicals that would cause renal damage.

Finally, studies in rats show that the mechanism of hepatotoxicity is different from nephrotoxicity, and the latter could be induced by a glutathione-conjugated paracetamol. In patients who already developed liver and renal failure might be present in the context of a hepatorenal syndrome.

It is known that gender is not a factor that could favour the onset of acute kidney injury in acetaminophen overdose and/or poisoning, however it is most common in children and adolescent. Other factors that could deplete the glutathione include taking concomitant nephrotoxic and/or hepatotoxic medications, high and recurrent doses of acetaminophen or the pre-existing renal disease. ${ }^{6,7}$ None of these factors were present in a patient presented in first case while in the second case frequent doses of paracetamol with concomitant administration of lormetazepam which mainly metabolized by liver could have been the trigger process.

The elevation of serum creatinine levels generally occurs from the second day after ingestion of paracetamol, with its peak at 5-7 days, which is followed by a gradual decline. This typical pattern of serum creatinine levels was seen in first case. In second case, there were high serum creatinine levels from baseline due to sustained overdosing of acetaminophen. As urea synthesis is impaired in acute liver failure, it is not an accurate guide to assess the severity of renal dysfunction by serum urea levels. Treatment of acetaminophen-induced kidney damage is usually supportive and symptomatic, requiring haemodialysis sessions in less than $10 \%$ of cases. It can take up to several weeks for complete resolution even when the liver function is completely restored. $\mathrm{N}$-acetylcysteine is not useful as an antidote to reverse the nephrotoxicity, and neither there is evidence that its use influence the normal of renal function recovery after 1-4 weeks.

The clinical pharmacist practicing in poison control centres should aware that renal manifestations can occur in acetaminophen poisoning and maximal renal injury lags beyond peak liver injury, and recovery is also more protracted. Therefore, assessment of renal function is mandatory in paracetamol poisoning.

\section{ABBREVIATIONS USED}

WHO: World Health Organization; AST: Aspartate transaminase; ALT: Alanine transaminase; LFT: Liver function test; IU/I: International unit/liter; NAPQI: Nacetyl-p-benzoquinoneimida.

\section{ABOUT AUTHORS}

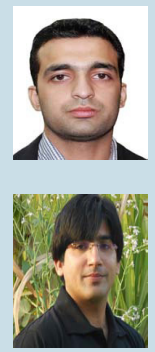

Faizan Mazhar: Is an academic and clinical pharmacist working at King Fahd Military Medical Complex, Dhahran, Saudi Arabia. He is a Board Certified Pharmacotherapy Specialist (BCPS) from the board of Pharmacy specialties (BPS), Washington. He holds a M. Phil degree in Clinical Pharmacology. He is also pursuing Ph.D. from University of Karachi, Pakistan. His practice and research specialty areas include infectious disease, geriatrics and medication safety.

Shahzad Akram: Is an in-patient pharmacist working in pharmaceutical care department at King Abdulaziz Medical City, National Guard Health Affairs, Riyadh, Saudi Arabia. He is a Board Certified Pharmacotherapy Specialist (BCPS) from the board of Pharmacy specialties (BPS) Washington. He has more than five years experience in clinical pharmacy. His practice and research specialty areas include infectious disease, pediatrics, and medication safety.

\section{REFERENCES}

1. Khandelwal N, James LP, Sanders C, Larson AM, Lee WM. Unrecognized acetaminophen toxicity as a cause of indeterminate acute liver failure. Hepatology. 2011;53(2):567-76.

2. Lines SW, Wood A, Bellamy MC, Lewington AJ. The outcomes of critically ill patients with combined severe acute liver and kidney injury secondary to paracetamol toxicity requiring renal replacement therapy. Renal failure. 2011;33(8):785-8.

3. Mazer M, Perrone J. Acetaminophen-induced nephrotoxicity: pathophysiology, clinical manifestations, and management. Journal of Medical Toxicology. 2008;4(1):2-6

4. Bachar S, Mahmud Z, Qais N. Antioxidant and hepatoprotective activities of ethanolic extracts of leaves of Premna esculenta Roxb. against carbon tetrachlo- ride-induced liver damage in rats. Journal of Young Pharmacists. 2012;4(4):228-34

5. Stern ST, Bruno MK, Horton RA, Hill DW, Roberts JC, Cohen SD. Contribution of acetaminophen-cysteine to acetaminophen nephrotoxicity II. Possible involvement of the $\gamma$-glutamyl cycle. Toxicology and applied pharmacology. 2005;202(2):160-71

6. von Mach M-A, Hermanns-Clausen M, Koch I, Hengstler J, Lauterbach M, Kaes $\mathrm{J}$, et al. Experiences of a poison center network with renal insufficiency in acetaminophen overdose: an analysis of 17 cases. Clinical Toxicology. 2005;43(1):31-7.

7. Akca S, Süleymanlar I, Tuncer M, Ersoy F, Süleymanlar G. Isolated acute renal failure due to paracetamol intoxication in an alcoholic patient. Nephron. $1999 ; 83(3): 270-1$ 